\title{
Repères
}

\section{Sélection thématique de livres}

Cette rubrique signale les ouvrages récemment parus en reprenant la quatrième de couverture ou la présentation des éditeurs. Les livres sont classés en trois catégories : les ouvrages thématiques par objet d'étude ; les ouvrages sur la science et ses rapports avec la société ; les ouvrages de réflexion sur les sciences ${ }^{1}$.

\section{Agriculture, alimentation}

\section{Penser et agir en agronome :} hommages à Michel Sebillotte Jean Boiffin, Thierry Doré (Eds) Quæ, 2012, 264 p., $38 €$

Michel Sebillotte a été une personnalité marquante du monde agronomique au cours des dernières décennies. Pédagogue brillant à la pensée intarissable, leader charismatique et visionnaire, il a contribué à refonder les bases de l'agronomie et a ouvert des perspectives de grande portée pour la recherche agronomique et le développement agricole. Dans tous les domaines où il a œuvré, il a laissé un apport conceptuel et méthodologique majeur, et fondé des communautés de travail à la fois durables et fécondes. Cet ouvrage rassemble les contributions de plusieurs dizaines d'auteurs qui ont été ses élèves, collègues et amis. Il est composé de textes d'analyse sur les apports de M. Sebillotte à l'enseignement et à la recherche en agronomie, au développement agricole, à la prospective, au développement régional et, plus globalement, aux orientations stratégiques de la recherche agronomique, ainsi que de témoignages et points de vue plus particuliers, et parfois plus personnels. Au-delà de l'hommage à une personnalité marquante et attachante, ces textes apportent un éclairage inédit sur cinquante années d'évolution de la discipline, de la recherche agronomique et du développement agricole en France.

Organisations et sociétés paysannes :

une lecture par la réciprocité

Éric Sabourin

Quæ, 2012, 282 p., $36 €$

Cet ouvrage de synthèse traite de l'application de la théorie de la réciprocité aux transformations des organisations et sociétés paysannes, en particulier dans leurs interactions avec l'État et les marchés; il s'appuie sur des études de cas menées depuis 30 ans dans quatre continents. La première partie présente la construction de la théorie de la réciprocité en sciences sociales, ses limites, son champ d'application et les critiques qui lui sont adressées, notamment dans le cadre du paradigme du don. La deuxième porte sur l'analyse du principe de réciprocité dans les organisations, communautés et sociétés paysannes, et sur sa confrontation avec la théorie de la gouvernance des communs, avec les théories de l'économie morale et de l'économie de l'affection. À partir d'exemples de réciprocité dans les sociétés rurales africaines, l'auteur met en évidence la permanence structurante de ces pratiques en milieu paysan dans d'autres continents. Dans la troisième partie, il analyse les relations de réciprocité dans les transactions de redistribution et de commercialisation, en particulier dans les marchés de proximité ou les filières courtes, ce qui conduit à un dialogue avec la sociologie économique et l'économie solidaire. Les formes d'aliénation spécifiques de la logique de réciprocité (asymétrie, clientélisme et paternalisme) sont abordées en fin d'ouvrage, en dialogue avec la sociologie politique.

La Terre : une marchandise?

Agriculture et mondialisation capitaliste

Thierry Pouch (Ed.)

L'Harmattan, 2012, 316 p., $32 €$

Dans la crise actuelle de la mondialisation, l'agriculture est redevenue un objet d'étude, voire une priorité pour certaines institutions internationales. La crise est bien une force de rappel indiquant que le secteur agricole demeure essentiel dans le fonctionnement et le devenir des sociétés. Plusieurs problématiques en découlent. L'agriculture est-elle en voie d'être absorbée par le

\footnotetext{
1 Cette réorganisation de la rubrique en grands champs thématiques est pour l'instant en cours d'évaluation et donc susceptible d'évolution.
} 
capitalisme mondialisé ou bien y a-t-il des foyers de résistance laissant entendre que la petite exploitation agricole a encore un avenir ? Les articles composant cet ouvrage apportent des éléments de réponse à cette question fondamentale. Les approches y sont à la fois internationales, nationales et régionales.

\section{Changement climatique}

Valuing Climate Change Mitigation: Applying Stated Preferences in the Presence of Uncertainty

Sonia Akter, Jeff Bennett

Edgar Elgar, 2012, 192 p., £65.00 (hardback)

This enriching book presents a holistic overview of climate change uncertainty and offers a number of pathways that could be used to account for such uncertainties in the stated preference valuation research. It shows that uncertainty plays an important role in determining the values of climate change mitigation benefits and, as the authors say: "If this uncertainty remains unaccounted for, there is a potential danger that the estimated economic values will misrepresent social preferences for public policy interventions to manage environmental externalities". It treats climate change using three dimensions of uncertainty: scenario, policy and preference. Conceptual frameworks are advanced to account simultaneously for these various dimensions of uncertainty. The authors then explore the impact of introducing these uncertainties into benefit estimates for the Australian Carbon Pollutions Reduction Scheme.

\section{Développement durable}

\section{Learning for Sustainability in Times of Accelerating Change \\ Arjen E.J. Wals, Peter Blaze Corcoran (Eds) \\ Wageningen Academic Publishers, 2012, 550 p., $€ 69.00$ (hardback)}

We live in turbulent times; our world is changing at accelerating speed. Information is everywhere, but wisdom appears in short supply when trying to address key inter-related challenges of our time such as: runaway climate change, the loss of biodiversity, the depletion of natural resources, the on-going homogenization of culture, and rising inequity. Living in such times has implications for education and learning. This book explores the possibilities of designing and facilitating learningbased change and transitions towards sustainability. In
31 chapters contributors from across the world discuss (re)emerging forms of learning that not only assist in breaking down unsustainable routines, forms of governance, production and consumption, but also can help create ones that are more sustainable. The book has been divided into three parts: re-orienting science and society, re-connecting people and planet and re-imagining education and learning. This is essential reading for educators, educational designers, change agents, researchers, students, policymakers and entrepreneurs alike, who are concerned about the well-being of the planet and convinced of our ability to do better.

\section{Normaliser au nom du développement durable \\ Pierre Alphandéry, Marcel Djama, Agnès Fortier, Ève Fouilleux (Eds) \\ Quæ, 2012, 200 p., $38 €$}

Dans le contexte actuel de globalisation néolibérale et de mise en cause de ses effets négatifs, l'objectif de cet ouvrage est de montrer que les dispositifs de normalisation ne sont pas seulement de nouvelles formes de régulation, d'institutions et de pratiques sociales, mais qu'ils renvoient en réalité à des enjeux éminemment politiques et à la construction de rapports de force spécifiques entre acteurs. Les différentes études de cas rassemblées ici portent sur des aires géographiques variées et s'appliquent aux secteurs agricole, agroalimentaire et forestier, qui demeurent parmi les plus dynamiques en matière de normalisation.

\section{Les défis énergétiques du $\mathrm{XXI}^{\mathrm{e}}$ siècle : transition, concurrence et efficacité au prisme des sciences humaines \\ Yves Bouvier (Ed.) \\ Peter Lang, 2012, 113 p., 24,90€}

En ces temps de transition énergétique, la question des nouvelles énergies et l'introduction de schémas économiques concurrentiels renouvellent les approches en termes de lieux de production, de comportements des citoyens-consommateurs et de recherche-développement. Pourtant, derrière l'apparente unité du terme " énergie », se trouve un produit stratégique aux sources et aux formes particulièrement variées. La tradition enseigne des dominations successives (bois-vent-hydraulique; charbon; hydrocarbures ; électrification), alors que le bilan énergétique a toujours été diversifié. La connaissance des énergies, dont le développement et le renouvellement au cours de ces dernières années sont manifestes, notamment dans les sciences humaines et sociales, ne progresse pas nécessairement au même rythme d'une source ou d'une forme à l'autre. Mais, de la disparité des approches, peut naître une dynamique : tel est le pari et l'enjeu des textes réunis dans ce volume. 


\section{Gestion des ressources}

\section{Collaborative Resilience: Moving Through Crisis to Opportunity}

Bruce Evan Goldstein (Ed.)

The MIT Press, 2011, 424 p., \$29 (paperback)

Drawing on recent work in the fields of planning and natural resource management, this book examines a range of efforts to enhance resilience through collaboration, describing communities that have survived and even thrived by building trust and interdependence. These collaborative efforts include environmental assessment methods in Cozumel, Mexico; the governance of a "climate protected community" in the Blackfoot Valley of Montana; fisheries management in Southeast Asia's Mekong region; and the restoration of natural fire regimes in U.S. forests. In addition to describing the many forms that collaboration can take - including consensus processes, learning networks, and truth and reconciliation commissions - the authors argue that collaborative resilience requires redefining the idea of resilience itself. A resilient system is not just discovered through good science; it emerges as a community debates and defines ecological and social features of the system and appropriate scales of activity. Poised between collaborative practice and resilience analysis, collaborative resilience is both a process and an outcome of collective engagement with social-ecological complexity.

\section{Rivières et rivaux : les frontières de l'eau}

Vazken Andréassian, Jean Margat

Quæ, 2012, 138 p., 29,50€

L'histoire des civilisations anciennes fourmille de rivalités liées à l'accès à l'eau, qui désaltère les assoiffés, irrigue les champs, fait tourner la roue des moulins, sert de fortification et matérialise les frontières. $Q u^{\prime}$ en est-il aujourd'hui ? Si, en Europe de l'Ouest, les affrontements ont laissé place à la coopération et à la paix, l'eau reste au cœur des grands enjeux mondiaux. C'est une ressource naturelle pas comme les autres. Vazken Andréassian et Jean Margat analysent les frontières physiques et humaines qui délimitent l'inégale répartition des eaux sur terre. D'où vient l'eau des rivières ? Par où passe la frontière fluviale? Comment inventorier les ressources en eau ? Des rivalités emblématiques liées à l'eau sont présentées pour des fleuves transfrontaliers comme le Tigre et 1'Euphrate, le Nil, le Jourdain, le Rhin, le Colorado, 1'Indus... Sont dévoilées les raisons qui rendent les conflits liés au partage de l'eau des rivières si complexes. Enfin, les auteurs examinent les raisons d'espérer, et de désespérer, au sujet de l'eau et des conflits qui pourraient concerner son partage équitable dans le futur.
Strategic Natural Resource Governance: Contemporary Environmental Perspectives /

La gouvernance des ressources naturelles : perspectives contemporaines dans le domaine de l'environnement Carmen Maganda, Olivier Petit (Eds)

Peter Lang, 2012, 241 p., $36 €$

Les ressources naturelles sont exploitées quotidiennement pour fournir des besoins élémentaires, mais elles sont également gérées pour différentes finalités. La notion de gouvernance environnementale stratégique a sans doute été l'une des notions qui ont rencontré le plus de succès, au niveau politique comme dans les milieux académiques, ces vingt dernières années, et un bilan s'avère aujourd'hui nécessaire. Cet ouvrage présente diverses approches de la gouvernance environnementale, visant notamment à comprendre le rôle des acteurs à différentes échelles, ce qui constitue un enjeu essentiel dans le domaine de l'environnement et des ressources naturelles. Il est destiné à un large lectorat composé de scientifiques, d'étudiants, mais aussi de gestionnaires de l'environnement opérant à toutes les échelles, du niveau local au niveau international.

\section{Le végétal saisi par le droit \\ William Dross (Ed.) \\ Bruylant, 2012, 280 p., $50 €$}

Le végétal est un enjeu majeur pour notre époque. L'accroissement de la population mondiale, qui atteint aujourd'hui sept milliards d'habitants, rend cruciale la question de sa capacité à trouver les ressources nécessaires à son alimentation. Le végétal, qu'il nourrisse directement ou indirectement l'homme, est au cœur de cette problématique de la sécurité alimentaire mondiale. Les réponses à ce défi ne se trouveront pas seulement dans les laboratoires de recherche : le droit a son mot à dire, car c'est précisément sa tâche première que d'instaurer la justice distributive, autrement dit de procéder à l'allocation des ressources entre les hommes. Les féroces batailles qui entourent la question de la brevetabilité du vivant montrent suffisamment le rôle de premier plan que le droit est appelé à jouer... Les contributions réunies dans cet ouvrage visent à rendre compte de la manière dont celui-ci appréhende le végétal. La question du brevet et celle, plus spécifique, du droit des obtentions végétales sont évidemment traitées. Mais il y a davantage, car, s'il faut garantir les droits de propriété intellectuelle, il faut aussi assurer le commerce international des denrées, protéger le végétal par la responsabilité civile et pénale; se soucier aussi de la manière dont la production agricole du moment n'hypothèque pas, du fait des pollutions induites, les capacités productives futures ; assurer, au travers d'une législation rurale adaptée, le maintien des capacités productives du pays... Tout cela en observant que le végétal ne nourrit pas seulement, mais qu'il peut aussi soigner. 


\section{Gestion et politiques de l'environnement}

\section{Environmental Democracy Facing Uncertainty}

Cécilia Claeys, Marie Jacqué (Eds)

Peter Lang, 2012, 185 p., 33,70€

This collective work provides a reflexive reading of environmental democracy as a new method of governance of the contemporary ecological issues that declining biodiversity, climate change and sustainable development present. The authors examine the links between the environment and democracy by questioning the status of actors, the manner of their involvement, the various ways of mobilising knowledge and the mechanisms of dialogue and decision-making based on study cases observed in different national contexts (Italy, France, Ireland, Germany, the Netherlands, Russia, Canada and Brazil). This international approach sheds light on the means of appropriation of environmental democracy on a local level and its ability to promote universal characteristics or to standardise the connection to the environment and politics.The originality of this work comes, among other things, from its transversality, associating texts with differing theoretical outlooks and methodology in an innovative way. Through this perspective ongoing processes of redefining environmental problems are revealed via the prisms of risks and uncertainty, thus assigning them a new role in aiding decision-making in a sociology that is in turn critical and committed.

\section{Géopolitique et environnement : les leçons de l'expérience malgache}

Hervé Rakoto Ramiarantsoa, Chantal Blanc-Pamard, Florence Pinton (Eds)

IRD Éditions, 2012, 296 p., $31 €$

La globalisation des politiques environnementales ne produit pas les mêmes effets dans tous les pays du Sud. Dans le même temps persiste la difficulté à penser de façon renouvelée les relations entre nature et société, entre conservation et développement, ce dont témoigne « Rio +20 », la conférence des Nations unies pour le développement durable tenue en juin 2012. Dans ce contexte, Madagascar, pays fortement engagé dans une démarche de conservation de son patrimoine forestier, s'avère un cas d'école emblématique. Quels acteurs contrôlent les modalités de production et de mise en œuvre des projets de valorisation de la forêt ? Par quelles institutions ces projets sont-ils financés ? Quelles retombées en termes de lutte contre la pauvreté peut-on en attendre? À travers un regard géopolitique partagé, les auteurs de cet ouvrage, issus de disciplines et d'horizons divers, interrogent le processus de construction des politiques environnementales et analysent les relations entre science, politique et société, alors que s'est progressivement imposée une vision de plus en plus marchande de la nature. Cette publication s'adresse à tous ceux - chercheurs, étudiants, praticiens du développement et décideurs - qui explorent pistes et retours d'expériences sur les relations entre géopolitique et environnement.

\section{Global Environmental Commons: Analytical and Political Challenges in Building Governance Mechanisms}

Éric Brousseau, Tom Dedeurwaerdere, Pierre-André Jouvet, Marc Willinger (Eds) Oxford University Press, 2012, 464 p., £63.00 (hardback)

Environmental challenges, and the potential solutions to address them, have a direct effect on living standards, the organization of economies, major infrastructures, and modes of urbanization. Since the publication of path-breaking contributions on the governance of environmental resources in the early 1990s, many political initiatives have been taken, numerous governance experiments have been conducted, and a large multi-disciplinary field of research has opened up. This interdisciplinary book takes stock of the knowledge that has accumulated to date, and addresses new challenges in the provision of environmental goods. It focuses on three essential dimensions with respect to governance. First, it addresses the issue of designing governance solutions through analyzing systems of rules, and levels of organization, in the governance and management of environmental issues. Second, it draws renewed attention to the negotiation processes among stakeholders playing a crucial role in reaching agreements over issues and solutions, and in choosing and implementing particular policy instruments. Finally, it shows that compliance depends on a combination of formal rules, enforced by recognized authorities, and informal obligations, such as social and individual norms. The evolution of the research frontiers on environmental governance shows that more legitimate and informed processes of collective decision, and more subtle and effective ways of managing compliance, can contribute to more effective policy. However, this book also illustrates that more democratic and effective governance should rely on more direct and pluralistic forms of involvement of citizens and stakeholders in the collective decision making processes.

\section{Patrimoines et développement durable : ressources, enjeux, lien social}

Nassima Dris (Ed.)

Presses universitaires de Rennes, 2012, 334 p., $20 €$

Loin de considérer les cultures locales comme une forme de repli ou de résistance au changement, cet ouvrage questionne les liens de proximité, les singularités locales et l'action publique dans leur interaction avec d'autres échelles du territoire. Il s'agit de mettre en lumière la place du patrimoine (bâti et naturel) dans le développement durable et la formation du lien social. Les 
contributions (sociologie, géographie, histoire, philosophie, architecture, urbanisme, anthropologie, ethnologie) abordent ces questions par la confrontation de champs théoriques et d'expériences locales situées en France et dans d'autres pays (Algérie, Cameroun, Canada, Italie, Maroc, Sénégal, Tunisie).

\section{Que faire des déchets ménagers?}

André Le Bozec, Sabine Barles, Nicolas Buclet, Gérard Keck

Quæ, 2012, 232 p., $37 €$

Comment organiser la gestion des déchets dans les prochaines années ? Quels sont les conflits entre la population et les décideurs? Comment les anticiper ? Quels sont les leviers économiques ? Comment intégrer les progrès sanitaires et environnementaux ? Une historienne de l'environnement, un urbaniste, un toxicologue et un ingénieur économiste répondent au questionnement des décideurs. Les techniques de gestion des déchets ménagers et les réglementations sont décrites en France et en Europe du Nord, et les coûts sont évalués et reliés au financement du service par la tarification incitative. Mais la gestion des déchets ménagers renvoie également aux modes de consommation, au devenir des biens usagés, aux impacts sur le milieu naturel et la santé publique. L'histoire de la gestion des déchets et l'analyse des risques sanitaires complètent ce panorama et l'exemple de la crise de la dioxine (1989-2000) montre la difficulté chronique à anticiper une crise sanitaire et à en accepter la dimension sociale. La prévention est l'enjeu majeur de la prochaine décennie, avec l'objectif de réduire la quantité d'ordures ménagères résiduelles en France à 100 kilos par habitant et par an. En association avec les citoyens, de plus en plus impliqués dans les services et la prévention, les décideurs sont conduits à élaborer des solutions techniques et organisationnelles acceptables et durables.

\section{La science en action}

La recherche, malade du management

Vincent de Gaulejac

Quæ, 2012, 96 p., 8,60€

La révolution managériale dans les institutions publiques, en lien avec la Révision générale des politiques publiques, modifie en profondeur le rapport au travail et l'activité des personnels. La recherche du résultat, l'avancement au mérite, le management par projet, l'obsession évaluatrice, produisent des tensions entre la culture du service public et la culture d'entreprise. Quels sont les paradigmes qui sous-tendent cette nouvelle gestion publique? Comment analyser les effets psycholo- giques, idéologiques et organisationnels de ces pratiques managériales ? Comment agir face à ce mal-être au travail ? L'auteur examine en quoi ces questions concernent aujourd'hui le monde de la recherche. Il s'interroge sur le glissement qui s'opère dans l'évaluation de la recherche quand l'excellence est définie et mesurée quantitativement. Au-delà de la communauté scientifique, directement concernée, cet ouvrage s'adresse à toute personne curieuse des relations entre les secteurs public et privé. En favorisant la réflexion et l'analyse, l'auteur souhaite appeler à une résistance citoyenne, raisonnée et argumentée.

Systèmes complexes : une introduction par la pratique Philippe Collard, Sébastien Verel, Manuel Clergue

Presses polytechniques et universitaires romandes, 2013, 306 p., 44,50€

Chacun peut observer dans le monde réel l'omniprésence de systèmes complexes constitués de nombreux éléments en interaction et dont les caractéristiques globales ne peuvent se réduire à celles de leurs composants : ce sont, par exemple, les cellules d'un organisme vivant, les colonies de fourmis, les écosystèmes ou encore les systèmes économiques, l'Internet et les réseaux sociaux. Dans le futur, nos sociétés devront relever l'immense défi scientifique et technologique qui consiste à comprendre ces systèmes pour les adapter, les contrôler et les modéliser. Au travers d'exemples choisis sans complication inutile, les auteurs proposent une introduction par la pratique aux systèmes complexes. Leur méthode repose sur la conviction que toute compréhension suppose un acte d'expérience ; l'ouvrage est composé d'ateliers où il s'agit de simuler une dynamique collective à partir de sa modélisation orientée agent. Les étudiants, les enseignants, les créateurs et, plus largement, toute personne avide de développer sa culture et d'actualiser ses connaissances tireront parti de cet ouvrage pluridisciplinaire accessible à un large public.

\section{Rapports sciences-technologies-société}

L'Homme simplifié : le syndrome de la touche étoile Jean-Michel Besnier

Fayard, 2012, 208 p., $18 €$

La mécanisation de l'humain a marqué le début des temps modernes. L'extension des technologies dites intelligentes consacrera-t-elle son aspiration à la bêtise ? "Appuyez sur la touche étoile », répète le serveur vocal qui contraint son interlocuteur à faire la bête pour être servi. Si les machines prétendent nous simplifier la vie, elles réduisent aussi nos comportements à la logique de leur fonctionnement dépourvu d'ambiguïté, d'ironie ou 
d'émotions. Parce qu'elle est insidieuse, la déshumanisation est redoutable. Jean-Michel Besnier dissèque ici sans ménagement «l'homme simplifié » que nous consentons à devenir, au gré des conceptions scientifiques et des innovations techniques. Étonnante, cette servitude volontaire appelle une révolte d'un nouveau style, que seule attiserait encore la littérature : celle de l'homme revendiquant sa complexité et son intériorité comme le signe de sa liberté.

\section{Sciences humaines et sociales}

\section{Géographie rurale et interdisciplinarité}

Philippe Madeline (Ed.)

Presses universitaires de Caen / MRSH, 2012, 344 p., $19 €$

Quelles sont les relations qu'entretient la géographie rurale avec les autres sciences sociales, y compris les sciences agronomiques, pour mieux appréhender les campagnes d'aujourd'hui ? Telle est la question que pose ce dernier numéro de la série «Enquêtes rurales ». Les contributions s'inscrivent dans un triple contexte. En premier lieu, la géographie rurale a tiré parti de rencontres plus ou moins fructueuses avec l'histoire, la sociologie, l'ethnologie, etc. À la lumière de ces expériences d'un passé proche ou lointain, comment s'effectuent les nouvelles collaborations? Par ailleurs, l'essor des campagnes multifonctionnelles a fait voler en éclats les repères d'une catégorie spatiale bien établie, celle $\mathrm{d}^{\prime}$ « espace rural » : dans ce contexte changeant, au sein duquel le secteur agricole s'est rétracté, la position des géographes a moins d'évidence. L'ouverture aux autres disciplines est davantage revendiquée ; mais que peuvent-ils en attendre? L'approche interdisciplinaire impose à chaque science sociale de se définir vis-à-vis de ses partenaires par ses angles d'étude, ses méthodes et ses concepts. Dans ce cadre, quelle est la contribution spécifique de la géographie à la connaissance des espaces ruraux ? Enfin, le contexte institutionnel lui-même favorise l'essor de contrats de recherche affichant des collaborations croisées. Au total, cet élargissement des champs disciplinaires mobilisés conduit-il à une meilleure compréhension de l'espace des sociétés? 